Praxis wären interindividuelle Unterschiede zwischen den Patienten interessant - im Sinne eines Stepped-Care-Modells ist anzunehmen, dass nicht alle Patienten eine $\mathrm{Zu}$ satztherapie benötigen. Daher gilt es herauszufinden, für wen vier Sitzungen KVTI ausreichend sind, und wer eine Augmentation benötigt. Eine weitere Limitation der Studie von Wong et al. ist, dass zwar Schlaf und komorbide Symptome umfangreich erfasst wurden, die Effektivität der Behandlung zur
Verbesserung der Lebensqualität jedoch nicht evaluiert wurde. Eine aktuelle Studie unserer Arbeitsgruppe konnte zeigen, dass die Akzeptanz- und Commitment-Therapie (ACT), ein Verfahren, das neben Achtsamkeitsmeditation auch die Klärung persönlicher Lebensziele und wertebasiertes Handeln umfasst, die Lebensqualität bei Nonrespondern auf KVTI verbessern kann [2].

Dr. med. Lukas Frase, Dipl.-Psych. Elisabeth

Hertenstein, Prof. Dr. med. Christoph Nissen

\section{Narkolepsie nach Schweinegrippe - autoimmun, postinfektiös oder statistischer Fehler?}

Die post-infektiöse Häufung von Narkolepsiefällen nach einer H1N1-Infektion lässt sich mit einem neu identifizierten Pathomechanismus erklären. Unklar ist, ob dies auch Konsequenzen für die Influenza-Impfung hat.

m Rahmen der Schweinegrippe-Pandemie mit Influenza H1N1 in den Jahren 2009/2010 wurden weltweit vereinzelte, aber insgesamt gehäuft Fälle der seltenen Schlaferkrankung Narkolepsie berichtet. Bei dieser Erkrankung führt ein Untergang orexinerger Neurone im Hypothalamus zu einer Instabilität der SchlafWachregulation mit gestörtem Nachtschlaf, exzessiver Tagesschläfrigkeit mit plötzlichen Einschlafattacken sowie spezifischen Phänomenen wie Kataplexien. Etwa 20 Fälle von Narkolepsie wurden zwischen 2010 und $2012 \mathrm{im}$ Kontext von Impfungen gegen $\mathrm{H} 1 \mathrm{~N} 1$ berichtet. In der Folge entstand eine teils hitzig geführte Debatte, über Nutzen und Risiken der Impfung sowie über die statistische Relevanz und Interpretation der Berichte. Ein vermuteter autoimmuner Mechanismus gegen körpereigene Orexine konnte bisher nicht bestätigt werden.

Vor diesem Hintergrund und der Tatsache, dass manche Influenzastämme direkte Schäden an zentralnervösen $\mathrm{Ner}$ venzellen hervorrufen können, berichtet ein internationales Team um Tesoriero [1] nun von narkolepsieähnlichen Störungen des Schlaf-Wach-Rhythmus durch eine Infektion mit H1N1 im Tiermodell. Hierfür wurden Mäuse, die durch Gendeletion keine B- und T-Zellen aufwiesen und deshalb nicht zu einer MHC-abhängigen Immunreaktion auf das Virus fähig wa- ren, intranasal mit H1N1 oder Kochsalz infiziert. Der Schlaf-Wach-Rhythmus wurde mittels EEG, EMG und der Aufzeichnung von Körperbewegungen erfasst. Nach vier Wochen traten bei den infizierten Mäusen im Vergleich zu den Kontrollen eine signifikante Fragmentierung des Nachtschlafs sowie sehr kurze REM-Latenzen (sleep onset REM) auf. Zudem kam es ebenfalls in Woche vier zu immer mehr Schlafphasen während der ansonsten weitestgehend aktiv und wach zugebrachten Phase. Zeitgleich wurde eine Zunahme des Theta-Bandes in der EEG-Spektralanalyse berichtet, was ebenfalls von Orexin-Knockout-Tiermodellen beschrieben ist. Eine anschließende immunhistochemische Untersuchung von Gehirnschnittpräparaten demonstrierte neben anderen Arealen einen Befall trigeminaler und olfaktorischer Nervenfasern als mögliche Eintrittspforte sowie orexinerger und Melatonin-concentrating-hormone-( $\mathrm{MCH}-)$ haltiger Neurone in verschiedenen hypothalamischen Kerngebieten, wobei vor allem orexinerge Neurone einen Untergang zeigten. Die Autoren interpretieren diese Daten als Nachweis einer direkten Schädigung hypothalamischer Neurone durch H1N1 mit der konsekutiven Entwicklung narkolepsieähnlicher Symptome. Durch die Verwendung immuninkompetenter Mäuse erscheint die Alternativhypothese
1. Wong MY, Ree MJ, Lee CW. Enhancing CBT for chronic insomnia: A randomised clinical trial of additive components of mindfulness or cognitive therapy. Clin Psychol Psychother 2015. Epub ahead of print. doi: 10.1002/ cpp.1980.

2. Hertenstein E et al. Quality of life improvements after acceptance and commitment therapy in nonresponders to cognitive behavioral therapy for primary insomnia. Psychother Psychosom 2014; 83 (6): 371 -3

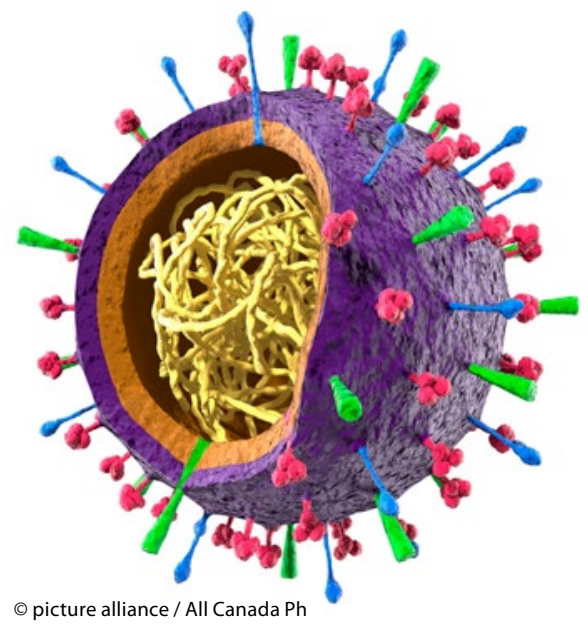

der Autoimmunvermittlung unwahrscheinlich.

\section{Kommentar}

In Anbetracht der breiten öffentlichen Diskussion im Kontext der H1N1-Impfung ist diese grundlagenwissenschaftliche Studie von großer Bedeutung. Die wissenschaftliche Identifizierung eines neuen Pathomechanismus anhand dessen eine postinfektiöse Häufung von Narkolepsiefällen nach einer H1N1-Infektion durch direkte Zellschädigung erklärbar ist, wirft viele neue Fragen auf. Möglicherweise kann ein besseres Verständnis des Infektionswegs helfen, durch immunmodulatorische Therapien die Abwehrfähigkeit gegenüber dem Virus zu stärken oder spezifische Risikopopulationen zu erkennen. Die Frage, ob ein ähnliches Muster bei der Impfung mit attenuierten Viren auftreten kann und welche Rolle die umstrittenen Wirkverstärker spielen, bleibt ungeklärt.

Dr. med. Lukas Frase, Dipl.-Psych. Elisabeth Hertenstein, Prof. Dr. med. Christoph Nissen

1. Tesoriero C et al. H1N1 influenza virus induces narcolepsy-like sleep disruption and targets sleep-wake regulatory neurons in mice. Proc Nat Acad Sci USA. 2016; 113 (3): E368-77 